\title{
Parasitoses em crianças na fase pré-escolar no Brasil: revisão bibliográfica
}

\author{
Parasitosis in preschool children in Brazil: bibliographic review \\ Parasitosis en preescolares en Brasil: revisión bibliográfica
}

Recebido: 15/12/2020 | Revisado: 23/12/2020 | Aceito: 27/12/2020 | Publicado: 02/01/2021

\author{
Danilo da Silva Munareto \\ ORCID: https://orcid.org/0000-0003-0860-1524 \\ Universidade Paranaense, Brasil \\ E-mail: danilomunareto@hotmail.com \\ Ana Paula Sokolowski de Lima \\ ORCID: https://orcid.org/0000-0002-5616-4528 \\ Universidade Paranaense, Brasil \\ E-mail: ana.sokolowski@edu.unipar.br \\ Giuliana Zardeto-Sabec \\ ORCID: https://orcid.org/0000-0003-1640-0714 \\ Universidade Paranaense, Brasil \\ E-mail: giulianazardeto@unipar.br \\ Suellen Laís Vicentino Vieira \\ ORCID: https://orcid.org/0000-0002-7169-3671 \\ Universidade Paranaense, Brasil \\ E-mail: suellen@prof.unipar.br
}

\begin{abstract}
Resumo
Estima-se que 200 milhões de crianças na idade pré-escolar de todo o mundo sejam afetadas por enteroparasitos, devido à falta, principalmente, de estratégias de melhoramento do nível socioeconômico da população, sendo assim, o objetivo deste trabalho foi realizar uma revisão de casos de parasitoses em criança na fase pré-escolar no Brasil. Para a realização do trabalho, foi feito um levantamento bibliográfico por meio de consulta eletrônica no banco de dados Scielo, Google acadêmico ${ }^{\circledast}$, PubMed e CAPES. Os estudos analisados demonstraram que os parasitas mais diagnosticados foram Ascaris lumbricoides, Giardia lamblia e Entamoeba histolytica na população infantil. Na ausência de sintomas iniciais, torna-se difícil o diagnóstico, porém os danos ao organismo em longo prazo a algum momento se manifestaram, como anemia, apatia, fadiga, sonolência excessiva, irritabilidade, entre outros, fazendo-se necessários exames de rotina. O exame parasitológico de fezes, que tem uma fundamental importância na identificação do parasita e também para o uso correto do medicamento, sendo ele uma vez especifico a cada parasitose. O uso indiscriminado de anti-helmínticos sem diagnóstico laboratorial pode desenvolver resistência medicamentosa, além de trazer um problema secundário ou adverso devido à exposição desnecessária ao medicamento. Diante do que foi exposto, conclui-se que as medidas de educação sanitária realizadas por professores e pais é uma das principais medidas de evitar as contaminações por parasitas intestinais em crianças. Fazem-se necessárias mais informações e trabalhos envolvendo crianças, pais e professores, no intuito de educar contra a infecção de parasitas humanos.
\end{abstract}

Palavras-chave: Anti-helmínticos; Menor de idade; Análises de solo; Anti-infecciosos.

\begin{abstract}
It is estimated that 200 million children of preschool age worldwide are affected by enteroparasites, mainly due to the lack of strategies to improve the population's socioeconomic level, therefore, the objective of this work was to carry out a review of cases of parasitic infections in preschool children in Brazil. To carry out the work, a bibliographic survey was made through electronic consultation in the Scielo database, Google academic, PubMed and CAPES. The analyzed studies showed that the most diagnosed parasites were Ascaris lumbricoides, Giardia lamblia and Entamoeba histolytica in the child population. In the absence of initial symptoms, diagnosis becomes difficult, but long-term damage to the body has manifested itself at some point, such as anemia, apathy, fatigue, excessive sleepiness, irritability, among others, making routine examinations necessary. The parasitological examination of feces, which is of fundamental importance in the identification of the parasite and also for the correct use of the drug, once it is specific to each parasitosis. The indiscriminate use of anthelmintics without laboratory diagnosis can develop drug resistance, in addition to causing a secondary or adverse problem due to unnecessary exposure to the drug. In view of the above, it is concluded the health education measures carried out by teachers and parents is one of the main measures to prevent contamination by intestinal parasites in children. More information and work involving children, parents and teachers is necessary in order to educate against infection by human parasites.
\end{abstract}

Keywords: Anthelmintics; Minor; Soil analysis; Anti-infectives. 


\begin{abstract}
Resumen
Se estima que 200 millones de niños en edad preescolar en todo el mundo se ven afectados por enteroparásitos, principalmente por la falta de estrategias para mejorar el nivel socioeconómico de la población, por lo que el objetivo de este trabajo fue realizar una revisión de casos de infecciones parasitarias en niños en edad preescolar en Brasil. Para la realización del trabajo se realizó un levantamiento bibliográfico mediante consulta electrónica en la base de datos Scielo, Google académico ${ }^{\circledR}$, PubMed y CAPES. Los estudios analizados mostraron que los parásitas más diagnosticados fueron Ascaris lumbricoides, Giardia lamblia y Entamoeba histolytica en la población infantil. Ante la ausencia de síntomas iniciales, el diagnóstico es difícil, pero en algún momento se ha manifestado daño a largo plazo en el organismo, como anemia, apatía, fatiga, somnolencia excesiva, irritabilidad, entre otros, haciendo necesarios los exámenes de rutina. El examen parasitológico de las heces, que es de fundamental importancia en la identificación del parásita y también para el correcto uso del fármaco, una vez que sea específico para cada parasitosis. El uso indiscriminado de antihelmínticos sin diagnóstico de laboratorio puede desarrollar farmacorresistencia, además de causar un problema secundario o adverso por la exposición innecesaria al fármaco. En vista de lo anterior, se concluye que las medidas de educación para la salud llevadas a cabo por maestros y padres es una de las principales medidas para prevenir la contaminación por parásitas intestinales en los niños. Se necesita más información y trabajo que involucre a niños, padres y maestros para educar contra la infección por parásitas humanos.
\end{abstract}

Palabras clave: Antihelmínticos; Menor; Análisis de suelos; Antiinfecciosos.

\title{
1. Introdução
}

As enteroparasitoses são infecções causadas por parasitas intestinais, sendo em sua maioria desencadeadas por protozoários e helmintos. É considerado um problema socioeconômico relevante, principalmente nas regiões subdesenvolvidas e em desenvolvimento, estando normalmente ligada a falta de saneamento básico e na escassez de higiene, contribuído desta forma, na manutenção do ciclo biológico e na disseminação dos parasitas (Silva et al., 2018).

As condições do hospedeiro, parasita e do ambiente formam a tríade epidemiológica, desencadeada pela precariedade do saneamento básico, higiene, dificuldades econômicas e a falta de orientação, facilitando a disseminação das parasitoses intestinais (Zanotto et al., 2018). Por conta da negligência sanitária, aumenta a probabilidade de contaminação, uma vez que as principais formas de transmissão de parasitas ocorrem pela via oral-fecal, através da ingestão de alimentos contaminados com ovos ou cistos, sendo estes normalmente microscópicos (Mamus et al., 2008).

Estima-se que 200 milhões de crianças na idade pré-escolar de todo o mundo sejam afetadas por enteroparasitas, devido à falta principalmente de estratégias de melhoramento do nível socioeconômico da população (Muñoz-Antoli et al., 2018). A faixa de idade mais propícia à contaminação com parasitoses está relacionada a crianças de 0 a 12 anos de idade, pois frequentam creches ou escolas, estando mais susceptíveis devido ao hábito de levar objetos à boca, como forma de exposição, bem como, a imaturidade de higiene (Fonseca, Barbosa \& Ferreira, 2017).

Um dos principais problemas das parasitoses na idade infantil de 0 a 12 anos, está relacionado com o sistema imunológico, pois as crianças não possuem um sistema imune completo, sendo incapazes de combater os parasitas (Antunes \& Libardone, 2017). Dessa forma, o impacto que as parasitoses podem acarretar nas crianças, está relacionado com a espécie e poliparasitismo podendo causar assim, desordem na saúde (Auler et al., 2018).

Os parasitas mais comuns de serem encontrados no território brasileiro são: Ascaris lumbricoides, Trichuris trichiura, Ancilostomideos, Entamoeba histolytica, Giardia lamblia, Endolimax nana e Entamoeba coli (Caldeira et al., 2019). Algumas parasitoses podem ser assintomáticas e outras sintomáticas. Quando apresentam sintomatologia, esta é de acordo com as características de cada parasita, por exemplo, a Entamoeba histolytica pode apresentar sintomas como diarreia, dor abdominal, náusea, megacólon e abcesso no fígado, pulmão ou cérebro, é o agente responsável por causar amebíase intestinal e podendo evoluir a amebíase invasiva extraintestinal (Vieira et al., 2012).

$\mathrm{Na}$ infecção por Ascaris lumbricoides, as manifestações clínicas comuns são cólica, náusea, desconforto abdominal e durante a fase de larva migratória pode apresentar eosinofilia aumentada (Dall'Orso et al., 2014). Na tricuríase, causada pelo verme T. trichiura, podem ocorrer cólicas, diarreia e prolapso retal, já em pacientes diagnosticados com Ancilostomídeos, esses apresentam quadros clínicos de prurido na fase aguda, síndrome de Loeffler, geofagia e em exames hematológicos apresentam normalmente anemias microcítica e hipocrômica (Caldeira et al., 2019).

A sintomatologia dos parasitas G. lamblia, E. nana e E. coli normalmente são acompanhadas de diarreia aguda, dor abdominal, fezes esteatorréicas, náusea e vômitos, que pode ser diagnosticada com um exame parasitológico de fezes, realizado em laboratórios de análises clínicas e posteriormente tratado (Welch et al., 2019).

De acordo com Brasil (2010), o Brasil está entre os países com maior índice de parasitoses intestinais, sendo alta a prevalência em crianças de idade pré-escolares, devido aos hábitos inadequados, falta da conscientização dos pais e professores e a falha da prática de higiene (Zanotto et al., 2018). Sendo assim, a Organização Mundial da Saúde (OMS) recomenda a desparasitação semestral ou anual das crianças e adultos acompanhados com higienização e saneamento básico (Welch et al., 2019).

Diante do alto número de crianças em idade pré-escolares contaminadas por enteroparasitas intestinais e pela negligência da realização do exame parasitológico de fezes para a identificação do parasita, o objetivo deste trabalho foi de realizar uma revisão de casos de parasitoses em crianças na fase pré-escolar no Brasil. 


\section{Metodologia}

O presente trabalho trata-se de uma revisão bibliográfica, da qual utilizou-se os bancos de dados Scientific Electronic Library Online (Scielo), Google acadêmico ${ }^{\circledR}$, National Center for Biotechnology Information (PubMed), Periódicos da Coordenação de Aperfeiçoamento de Pessoal de Nível Superior (CAPES), bem como, bancos de teses e dissertações.

Foram utilizadas as palavras-chaves: parasitoses intestinais; enteroparasitoses; parasitoses infantis; parasitas intestinais; parasitoses em pré-escolares no Brasil. O presente estudo nos permite aprofundar, no âmbito da investigação de diversas referências, os discursos e os principais temas abordados, fazendo uma compilação que nos permite olhar para um mesmo objeto de investigação através de diferentes olhares (Pereira et al., 2018) no desenvolvimento do estudo, foram analisados artigos em inglês, português e espanhol dos últimos anos.

\section{Resultados e Discussões \\ 3.1 Enteroparasitoses}

As enteroparasitoses são doenças que possuem como agentes helmintos ou protozoários causadores de alguns tipos de infecção no trato gastrointestinal, acometendo principalmente países em desenvolvimento (Fonseca, Barbosa \& Ferreira, 2017). Apresentam baixo nível de mortalidade, porém altas taxas de morbidades, principalmente em crianças, levando a desnutrição e prejudicando seu desenvolvimento cognitivo e sendo considerado um grande problema de saúde pública (Astudillo \& Brava, 2017).

Dentro dos protozoários intestinais, os mais relevantes são E. histolytica, G. lamblia, E. coli e E. nana possuindo característica como:

Entamoeba histolytica: Possui tamanho de 5 a $20 \mu \mathrm{m}$, apresentam cisto imaturo de forma arredondada, com parede cística delicada, presença de um ou mais núcleos com cariossoma central, corpos cromatóides em forma de bastão ou charuto e ocasionalmente, presença de vacúolo de glicogênio, o cisto maduro com quatro núcleos e ausência de corpos cromatóides. Na forma de trofozoíto são irregulares, com membrana citoplasmática delicada, presença de um núcleo com cariossoma central e puntiforme com tamanho de $10 \mu \mathrm{m}$ a $60 \mu \mathrm{m}$ (Figura 1. A) (Almeida \& Leite, 2020).

Giardia lamblia: Apresenta cisto de forma ovalada, com parede cística delicada, presença de quatro núcleos com cariossoma central, corpos parabasais dispostos transversalmente e axonemas dispostos longitudinalmente com tamanho de 8 $\mu \mathrm{m}$ a $15 \mu \mathrm{m}$, o trofozoíto possui forma piriforme e simetria bilateral, com membrana celular delicada, presença de dois núcleos com cariossoma central, corpos parabasais e axonemas dispostos longitudinalmente, apresentam quatro pares de flagelos não visíveis com tamanho de $10 \mu \mathrm{m}$ a $24 \mu \mathrm{m}$ (Figura 1. B) (Berne et al., 2014).

Entamoeba coli: Possui cisto maduro de forma arredondada, com parede cística delicada, contendo até oito núcleos com cariossoma central com tamanho de $15 \mu \mathrm{m}$ a $25 \mu \mathrm{m}$, o trofozoíto de forma irregular, com membrana citoplasmática delicada, presença de um núcleo com cariossoma excêntrico e grosseiro e cromatina periférica formada por grânulos grosseiros e de distribuição irregular com tamanho de $18 \mu \mathrm{m}$ a $28 \mu \mathrm{m}$ (Figura 1. C) (Norberg et al., 2008).

Endolimax nana: Possui cistos arredondados com tamanho aproximado de $8 \mu \mathrm{m}$ a $10 \mu \mathrm{m}$, possui quatro núcleos e geralmente aparecem em grandes quantidades, os trofozoítos medem de $10 \mu \mathrm{m}$ a $12 \mu \mathrm{m}$ de tamanho, o núcleo apresenta cariossoma grande e irregular, central ou excêntrico, membrana nuclear fina e ausência de cromatina periférica na membrana nuclear (Figura 1. D) (Norberg et al., 2008).

Figura 1. Protozoários intestinais mais relevantes nas crianças de 0-12 anos de idade.

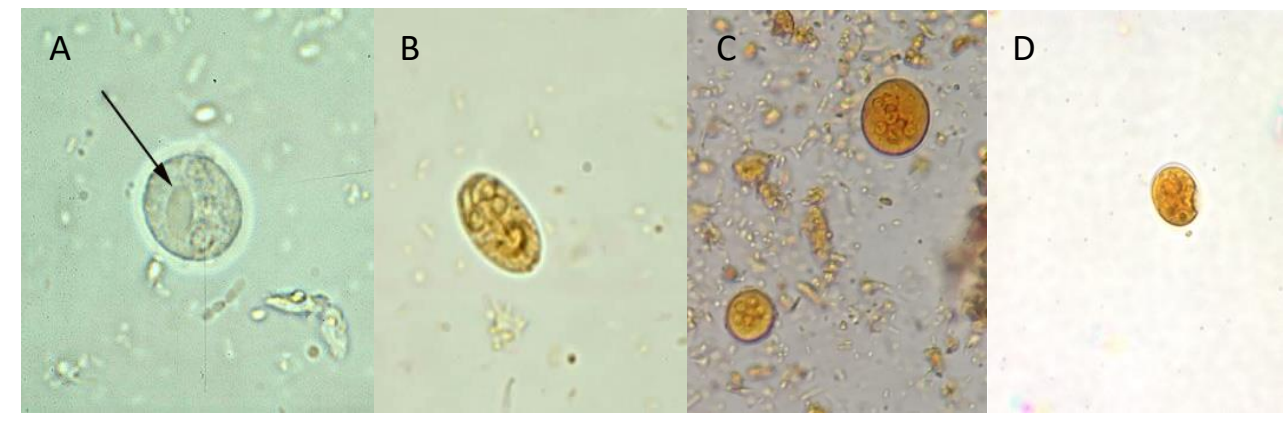

Legenda: A: cisto de E. histolytica; B: cisto de G. lamblia; C: cisto de E. coli; D: cisto de E. nana. Fonte: Autores (2020) adaptadas de CDC (2017).

Os helmintos são parasitas de vida livre, podendo ser encontrados em plantas, animais e no homem. Apresentam duas formas evolutivas principais, os ovos e os vermes adultos, além de fases intermediárias como as larvas dependendo do parasita (Mamus et al., 2008). Entre os vermes predominantes estão o Ascaris lumbricoides, Enterobius vermicularis e na forma 
infectante de larvas, Ancylostoma duodenale e Strongyloides stercoralis, aderindo principalmente ao intestino do homem (Siqueira \& Tristão, 2019).

Ascaris lumbricoides: Apresenta ovo fértil de formato oval ou quase esférico de casca espessa e célula ovo no interior, formada por três camadas apresentando em média, $60 \mu \mathrm{m}$ de comprimento, a camada mais externa recebe o nome de membrana mamilonada (Figura 2. A); possui ovo infértil de formato alongado, apresentando de $80 \mu \mathrm{m}$ a $90 \mu \mathrm{m}$ de comprimento, casca mais delgada com camada albuminosa muito reduzida, irregular ou ausente, no interior do ovo infértil, sendo cheio de grânulos refringentes, de aspecto grosseiro (Figura 2. B); o ovo decorticado tem formato oval ou quase esférico, apresentando camada interna e média, com célula ovo em seu interior, mas que perdeu sua membrana mamilonada (Figura 2. C); os vermes adultos são longos, cilíndricos e com extremidades afiladas, as fêmeas são maiores e mais grossas, tendo a parte posterior retilínea ou ligeiramente encurvada, os machos tem enrolamento ventral, espiralado na extremidade caudal (Figura 2. D) (Teixeira et al., 2018).

Enterobius vermicularis: As larvas (Figura 2. E) e os ovos (Figura 2. F) podem ser encontrados em amostras de fezes pela tamisação e em fraldas ou na região perianal, os ovos apresenta formato da letra "D", com $55 \mu \mathrm{m}$ x $25 \mu \mathrm{m}$ de tamanho, cor clara, casca espessa formada por membranas justapostas, em seu interior pode ser encontrado massa embrionária ou larva já formada (Lins et al., 2016). Larva adulta, mede cerca de $1 \mathrm{~cm}$ de comprimento, com corpo fusiforme, extremidades finas e cor esbranquiçada (Neves, Melo \& Genaro, 2011).

Ancylostoma duodenale/Necator americanus: Seu ovo apresenta tamanho variando de $0,8 \mathrm{~cm}$ a $1,3 \mathrm{~cm}$, possue forma ovalada, casca fina e transparente e um espaço largo e claro entre a casca e o conteúdo dos ovos (Figura 2. G), as larvas rabditóide apresentam bulbo esofagiano (esôfago do tipo rabditóide) e vestibulo bucal longo, já as larvas filarióide apresentam esôfago cilíndrico (do tipo filarióide) e cauda pontiaguda (Figura 2. H) (Fernandes et al., 2012).

Strongyloides stercoralis: Larva rabditóide mede aproximadamente 0,25 mm, possui esôfago rabditóide, vestíbulo bucal curto e primórdio genital visível, já a larva filarióide mede cerca de $0,5 \mathrm{~mm}$ e contém esôfago cilíndrico que se estende quase até a metade do corpo da larva, a fêmea é maior $(1,5 \mathrm{~mm})$ e possui extremidade posterior delgada e voltada ventralmente, apresentando duas espículas pequenas (Figura 2. I) (Fernandes et al., 2012).

Figura 2. Helmintos intestinais mais relevantes nas crianças de 0-12 anos de idade.

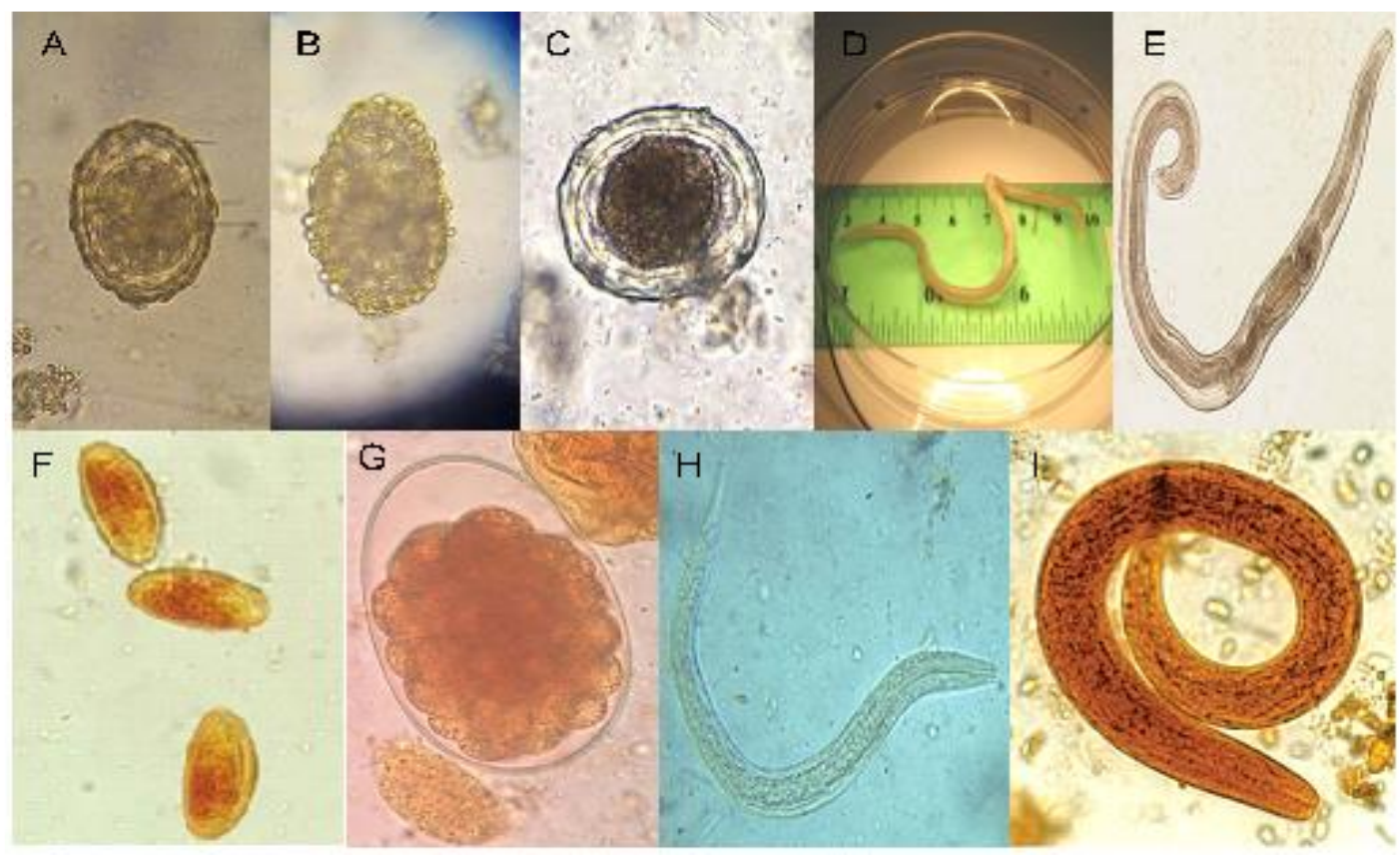


Legenda: A: ovo fértil de A. lumbricoides; B: ovo infértil de A. lumbricoides; C: ovo decorticado de A. lumbricoides; D: larva adulta de A. lumbricoides; E: larva de E. vermicularis; F: ovos de E. vermicularis; G: ovo de N. americanus; H: larva de A. duodenalis; I: larva de S. stercoralis. Fonte: Autores (2020) adaptadas de CDC (2017).

A prevalência das enteroparasitoses está diretamente relacionada a um conjunto de fatores como: saneamento básico, falta de educação sanitária, clima, hábitos de higiene pessoal e coletiva, como lavagem das mãos e alimentos irregulares, e de baixas condições socioeconômicas (Muñoz-Antoli et al., 2018).

Em crianças, os fatores se exacerbam, estando diretamente relacionados com hábitos precários. A partir dos primeiros meses de vida, a criança inicia o desenvolvimento das coordenações motoras e adquire aos poucos habilidades de pegar objetivos, levando-os à boca como forma de exploração e reconhecimento do mundo que o cerca (Astudillo \& Brava, 2017).

Devido à imaturidade de compreensão, bem como, de conhecimento com hábitos higiênicos, o fato de levar "tudo" à boca, eleva a chances de contaminação por parasitas, sendo assim a importância do conhecimento dos pais sobre as vias de transmissão e como prevenir, contribuindo na diminuição da taxa de parasitose nas crianças (Fonseca, Barbosa \& Ferreira, 2017).

\subsection{Epidemiologia}

A prevalência das parasitoses sofre variações no território brasileiro, devido às condições climática e socioeconômica de cada estado, estando diretamente relacionadas a ocorrências dos diferentes gêneros de enteroparasitas (Araújo et al., 2020).

Wiebbelling et al. (2019), relatam que 36\% da população brasileira apresentam algum tipo de parasitose intestinal, sendo as crianças mais susceptíveis, chegando a 55,3\% por falta de acesso básico como rede de esgoto, água tratada e conscientização dos pais sobre a higienização adequada. Estima-se que $50,26 \%$ da população brasileira não possui saneamento básico, representando cerca de 102 milhões de pessoas, aspecto importante que contribui para o aumento da contaminação (Bragagnollo et al., 2019).

A vulnerabilidade de crianças em idade escolar a parasitoses relaciona-se normalmente com a falta de higienização, mudanças comportamentais, desenvolvimento e mobilidade, possibilitando a interação com o meio ambiente, estando diretamente desta forma exposto a objetos contaminados e consequentemente aumentando a prevalência de infecções parasitárias nessa idade (Fonseca, Barbosa \& Ferreira, 2017). Já a diminuição da doença em jovens e adultos é explicada por possuírem resposta imunológica adaptativa e por modos de higiene mais eficaz comparada com as crianças (Falavigna et al., 2008). Sendo a educação sanitária uma das medidas de prevenção que tem se mostrado mais eficaz no combate das parasitoses (Zardeto-Sabec et al., 2020).

De acordo com uma análise integrativa de casos de parasitose no Brasil, os helmintos foram os mais prevalentes nas crianças estudadas de Seres (Goiás), sendo o A. lumbricoides (58,3\%) o mais predominante, apresentando uma prevalência de até $70 \%$ das parasitoses em crianças de idade escolar, por outro lado, o protozoário mais relatado no estudo é a G. lamblia com uma prevalência que variou de 4,9 a $96 \%$. Apesar de serem parasitas de comum distribuição no país, estes tornam-se negligenciados por falta de medidas de prevenção, passando despercebidos e tornando um grande problema de saúde pública (Solano-Barquero et al., 2018).

Ascaris lumbricoides possui um ciclo de vida que se desenvolve principalmente no corpo humano, podendo acometer o intestino, pulmão e fígado. A infecção ocorre com a ingestão dos ovos, que primeiramente chegam ao intestino delgado onde eclodem por conta do suco gástrico liberando a larva do seu interior, estas atravessam a parede do intestino chegando à circulação sanguínea podendo ir até o fígado. Posteriormente as larvas conseguem atingir o coração, sendo levadas pela artéria pulmonar até o pulmão podendo causar a síndrome de Loeffler, que é caracterizada por aumento da quantidade de eosinófilo no pulmão, bronquite e tosse. Em seguida, as larvas podem chegar aos brônquios e traqueia, em direção à epiglote, uma vez lá 
são deglutidos voltando ao intestino delgado assim repetindo o ciclo ou sendo liberados nas fezes (Figura 3) (Dall'Orso et al., 2014).

Figura 3. Ciclo de vida do Ascaris lumbricoides.

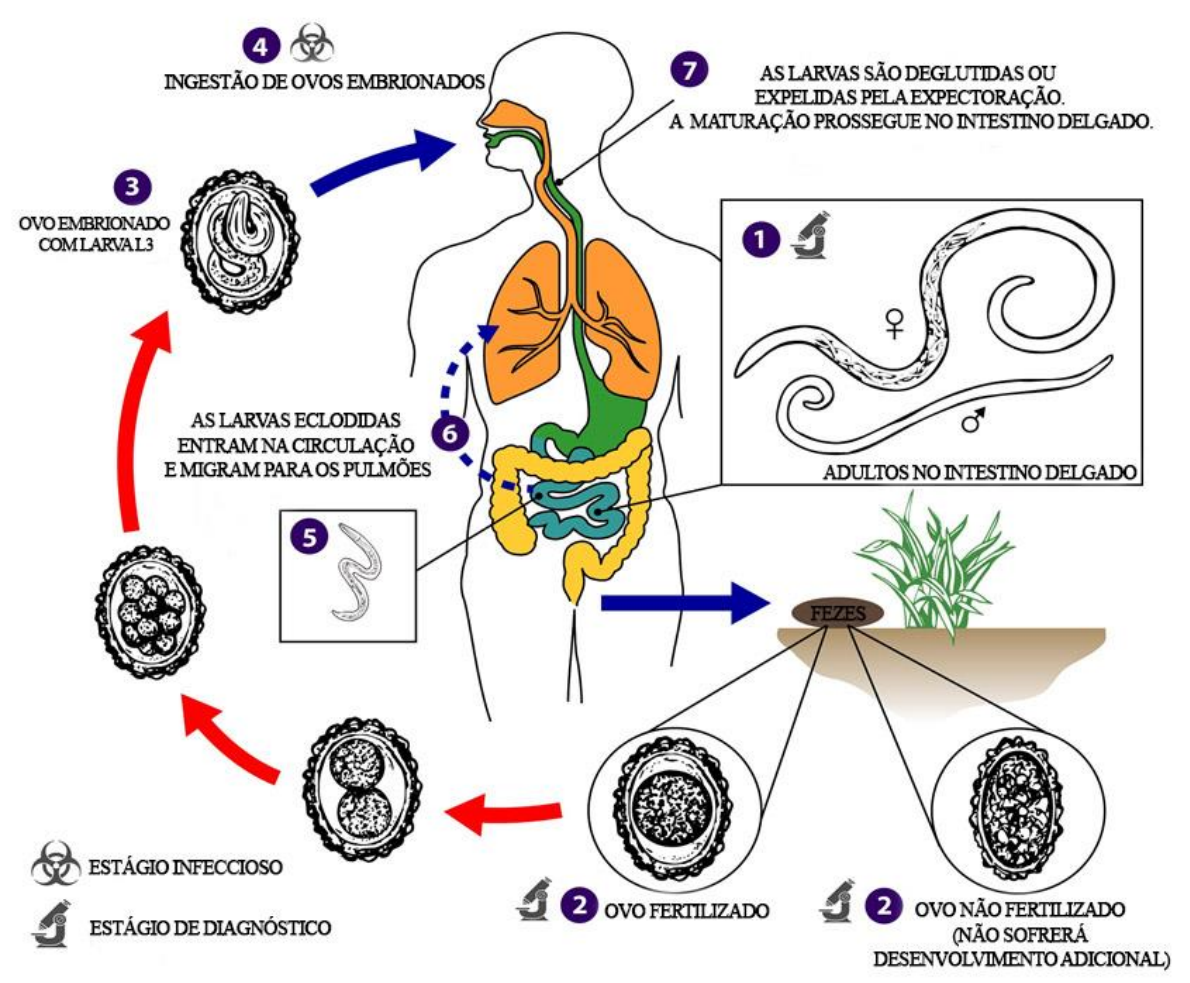

Fonte: Autores (2020) adaptada de CDC (2017).

Um estudo realizado por Siqueira e Tristão (2019), analisando a prevalência de contaminação de crianças de 0 a 12 anos em laudos parasitológicos de fezes de um laboratório de análises clínicas privado do município de Alto Paraíso (Rondônia) nos anos de 2017-2018, foi verificado o diagnóstico A. lumbricoides como o mais prevalente dentro da classe dos helmintos e entre os protozoários a E. histolytica e G. lamblia. O estudo ainda apontou que $47 \%$ das crianças de fase escolar, apresentaram alguma forma de parasita intestinal podendo ser encontrados cistos, ovos ou larvas de alguma espécie.

O ciclo biológico da E. histolytica é monoxênico, necessitando apenas de um hospedeiro para completar o ciclo (Figura 4). Apresenta quatro estádios evolutivos: cisto (forma infecciosa e não móvel), trofozoíto (forma invasiva e móvel), pré-cisto e metacisto, sendo as duas últimas formas intermédias (Castro et al., 2019). 
Figura 4. Ciclo de vida da Entamoeba histolytica.

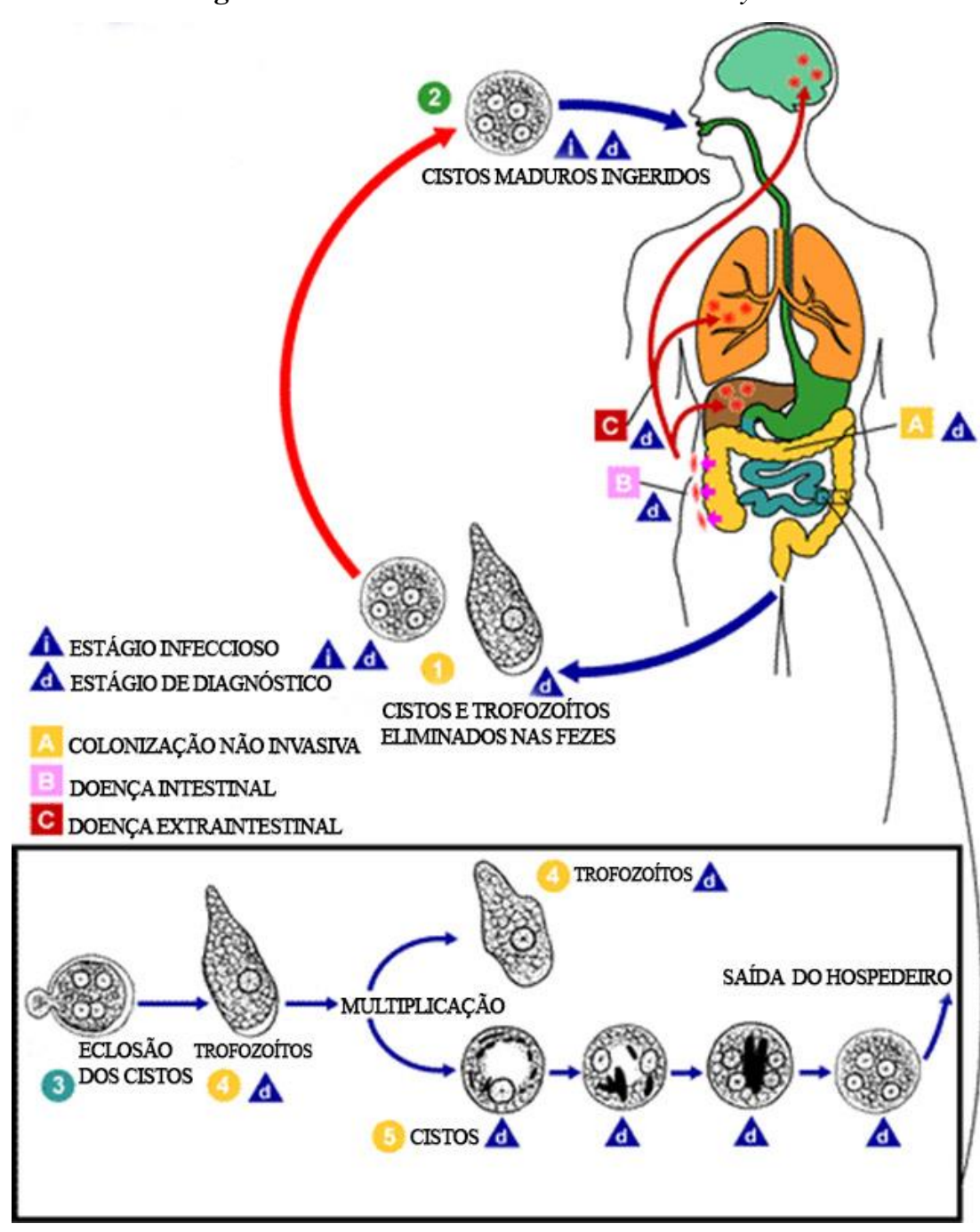

Fonte: Autores (2020) adaptada de CDC (2017).

São identificadas pelo menos oito amebas (E. histolytica, Entamoeba dispar, Entamoeba moshkovskii, E. coli, Entamoeba. hartmanni, Entamoeba polecki, Iodamoeba bütschlii e Endolimax. nana) que vivem no lúmen intestinal do ser humano, sendo que todas elas, com exceção da E. histolytica, são consideradas organismos comensais (não patogênicos e não invasivos) (Castro et al., 2019).

Em estudo realizado por Araújo et al. (2020), em crianças de uma creche pública no Sul do Brasil, com faixa etária de 3 a 6 anos, relataram a frequência de parasitoses intestinais de 18\%, sendo os cistos de G. lamblia (10\%) o parasita predominante, seguido de A. lumbricoides e Trichuris trichiura (6\%) e E. vermicularis (2\%). No mesmo estudo, foram encontrados que 43,9\% das crianças foram soropositivas para Toxocara spp, estando associado às condições sanitárias inadequadas como tratamento de água, coleta de lixo e assistência médica da cidade.

Giardia lamblia é um protozoário flagelado, a infecção ocorre através do ciclo que acontece após ingerir cistos contidos em água ou alimentos, estes quando chegam ao intestino delgado transformam-se em trofozoítos que sofrem divisão binária e chegam à luz do intestino onde podem ficar livres ou fixados na parede do intestino (Figura 5) (Lacerda \& Dias, 2017). 
Figura 5. Ciclo de vida da Giardia lamblia.

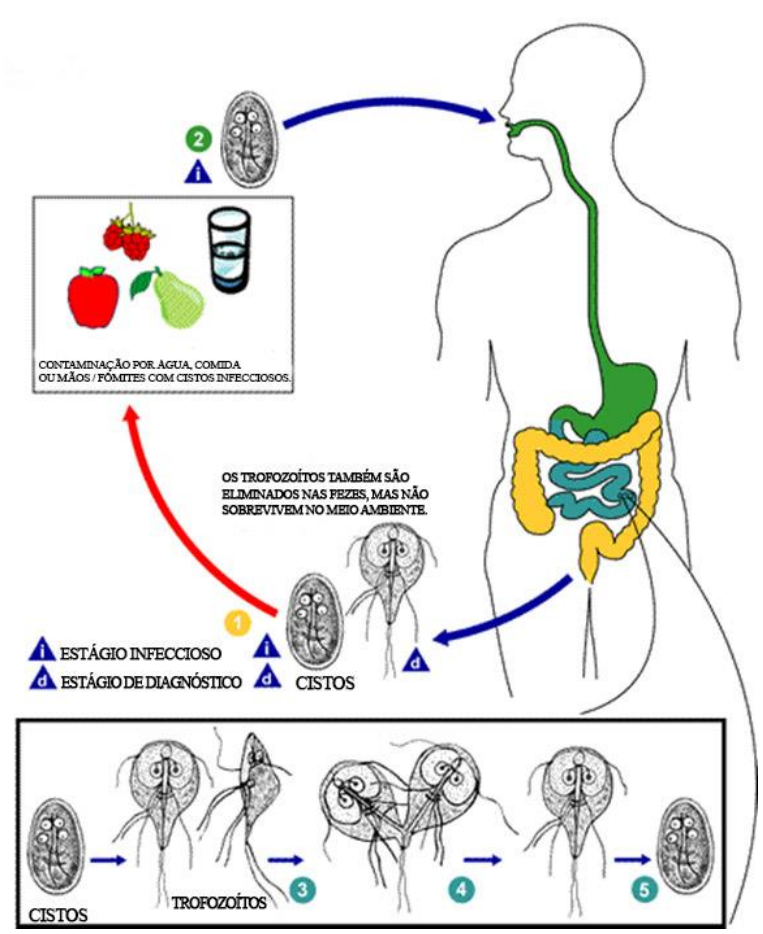

Fonte: Autores (2020) adaptada de CDC (2017).

Entretanto Fontes et al. (2017), analisaram áreas de lazer de escolas primárias e centros de educação infantil em São Mateus (Espírito Santo), e verificaram que 77\% das areias das regiões analisadas obtiveram resultados positivos para cistos de protozoários, ovos e larvas de helmintos, verificando a presença de Toxocara canis (66,7\%), A. lumbricoides (27,8\%) e Hymenolepis nana $(5,5 \%)$.

Em análise de laudos do exame parasitológico de fezes de um laboratório de análises clínicas da cidade de Umuarama (Paraná) do ano de 2018, foi realizado levantamento de dados que foi observado 3.333 laudos, sendo que a prevalência na idade de 0 a 10 anos é de 47,20\% que corresponde a 118 indivíduos infectados, verificou-se a maior prevalência de Endolimax nana $(39,16 \%)$ (Zardeto-Sabec et al., 2020).

O Brasil é um país com um clima variado, de questão socioeconômica desigual, saneamento básico falho, sendo estas causas favorecedoras para um maior número de pessoas infectadas por parasitas (Antunes; Libardoni, 2017). Além do mais, como as parasitoses intestinais não fazem parte de programas de notificação compulsória, estas enfermidades são na maioria das vezes negligenciadas (Zanotto et al., 2018).

O impacto clínico das enteroparasitoses pode ser desde assintomática até a presença de sintomas agudos como dores abdominais, diarreia, sonolência, indisposição, apatia, podendo evoluir para situações intensas como comprometimento do estado nutricional, como má absorção de nutrientes, hemorragias, anemias, diminuição do rendimento escolar e concentração (Cavalcante, Melo \& Lima, 2015).

A morbidade de parasitoses intestinais possui alta frequência em crianças e em pacientes imunossuprimidos (Villamizar et al., 2019). A idade pré-escolar e escolar é o principal fator de transmissão, por hábitos não higiênicos como geofagia, colocar mão na boca sem lavar corretamente antes ou depois de comer e brincar com animais, que podem transportar os parasitas (Cazorla-Perfetti, 2014). 
As creches possuem um papel importante na educação e desenvolvimento social da criança, juntamente com os pais ou responsáveis em alertar sobre a importância do cuidado e prevenção das parasitoses intestinais. A alta taxa de contaminação de crianças por enteroparasitoses intestinais está diretamente relacionada com a classe social, desenvolvimento socioeconômico, educação sanitária e sistema imunológico pouco desenvolvido (Araújo et al., 2020).

O déficit de infraestrutura nas cidades e falta de saneamento básico adequado, desencadeiam um destino incorreto das fezes e urina, o que aumenta a aparição de doenças infecciosas e o parasitismo em crianças, tornando um problema sanitário para o país (Serrano, 2018). Segundo Lacerda e Jardim (2017), uma das medidas de prevenção, está ligada às organizações governamentais e não governamentais em contribuir para a melhoria da problemática, trata-se de condições adequadas de saneamento como água tratada, rede de esgoto e educação sanitária.

A implantação de medidas profiláticas nas creches para professores, pais e crianças é de suma importância para rompimento da disseminação das parasitoses. Medidas educativas como palestras para pais e alunos, distribuição de folder na escola e creches da qual ilustra imagens e conta histórias sobre as parasitoses de forma educativa voltada à criança, são formas efetivas e da qual proporciona um aprendizado e interação entre pais, crianças e educadores (Andrade et al., 2019). Além do mais, tem-se no final do processo, normalmente, a conscientização dos adultos para as formas de prevenção das enteroparasitoses além da importância da realização do exame parasitológico de fezes e o tratamento correto (Wiebbelling et al., 2019).

A utilização de material educativo com temática de qualidade facilita chamar a atenção das crianças alvo, como folders educativos sobre parasitoses. Vale ressaltar que muitos materiais encontrados na internet não atingem a qualidade necessária para esclarecer a população sobre a problemática, muitas vezes não refletindo a realidade.

\subsection{Formas de diagnóstico}

Para o diagnóstico de parasitoses, a realização do exame parasitológico de fezes deve ser feito. A coleta do material fecal é realizada em frasco estéril, de tampa larga e rosqueável com uma quantidade mínima de 5 gramas de fezes. As amostras são analisadas macroscopicamente observando-se a consistência, se apresenta característica diarreica, e microscopicamente por métodos diretos em lâmina e métodos de concentração (Zanotto et al., 2018).

As técnicas de concentração utilizadas normalmente são de Hoffman, Pons e Janer ou Lutz, da qual é baseado na sedimentação espontânea em água, através da ação da gravidade, sendo eficaz para ovos e larvas de helmintos e cistos de protozoários, tornando-se principalmente útil para a pesquisa de ovos pesados (Zardeto-Sabec et al., 2020), a leitura poderá ser realizada a partir de 4 (quatro) horas após o preparo (tempo mínimo necessário para a sedimentação) até 72 (setenta e duas) horas (Neves, Melo \& Genaro, 2011; Zardeto-Sabec, 2019).

A técnica de Faust, que consiste na Centrífugo-Flutuação em Sulfato de Zinco. Normalmente utilizada para a pesquisa de cistos de protozoários e ovos leves de helmintos. A amostra deve ser centrifugada no mínimo duas vezes com água destilada, sendo que para melhorar a identificação dos cistos de protozoários, o recomendado é a centrifugação de três a quatro vezes, durante $1(\mathrm{um})$ minuto, clarificando significativamente a amostra. Após a última centrifugação com a água destilada, coloca-se em suspensão (sulfato de zinco a 33\%, densidade de $1,18 \mathrm{~g} / \mathrm{mL}$ ) no meio de flutuação, centrífuga-se por 1 (um) minuto a 2.500 RPM, após recolhe-se o sobrenadante com alça de platina e deposita-se em lâmina (Cardoso et al., 2018; Zardeto-Sabec, 2019), acrescenta-se 1 (uma) gota de lugol e lamínula, devendo a leitura ser imediata, pois o contato do parasita com o reagente por um longo período de tempo pode deformar as formas parasitárias, principalmente os cistos de protozoários (Neves, Melo \& Genaro, 2011).

O método de Ritchie consiste na sedimentação forçada para diagnóstico parasitário, porém apresenta como desvantagem o uso do éter etílico e formaldeído. No método direto, o exame ocorre pela deposição do material fecal entre 
lâmina e lamínula acrescido de lugol. O exame direto é indicado principalmente para fezes diarreicas, no intuito de se observar trofozoítos (Antunes; Libardoni, 2017), a leitura deverá ser imediata (Neves, Melo \& Genaro, 2011).

Para a pesquisa dos principais helmintos e protozoários intestinais em humanos, o exame direto a fresco é muito utilizado. O procedimento é colocar duas e três gotas de salina a 0,85\% em uma lâmina de microscopia; tocar, com a ponta de um palito, em vários pontos das fezes frescas, transferindo uma pequena porção destas para a lâmina; espalhar as fezes com a salina e observar no microscópio; pode-se corar com lugol (Neves, Melo \& Genaro, 2011; Zardeto-Sabec, 2019).

Esse método apresenta baixa sensibilidade, pois não utiliza um processo para a concentração das formas parasitárias, a quantidade de fezes empregada é muito pequena e o excesso de detritos pode mascarar as formas parasitárias. Estas serão detectadas quando presentes em grande quantidade. Entretanto, este método pode ser útil para a pesquisa de trofozoítos de protozoários em fezes diarreicas recém-emitidas (no máximo 30 minutos após). Sendo aconselhável examinar, no mínimo, três lâminas de cada amostra (Neves, Melo \& Genaro, 2011).

A utilização de dois ou mais métodos é útil para um diagnóstico preciso de infecção causada por parasitas intestinais, principalmente helmintos e protozoários (Batista, Trevisol \& Trevisol, 2009).

\subsection{Tratamento}

Os antiparasitários são fármacos que agem com o intuito de eliminar parasitas que estão localizados no trato gastrointestinal sendo ovo, cisto e protozoário que invadem o tecido (Andrade et al., 2011). O uso indiscriminado de antihelmínticos sem diagnóstico laboratorial pode desenvolver resistência medicamentosa, além de trazer um problema secundário ou adverso devido à exposição desnecessária ao medicamento, desta forma a realização do exame parasitológico de fezes, que tem uma fundamental importância na identificação do parasita, permite um uso correto do medicamento, sendo este uma vez especifico a cada parasita (Freitas, 2017).

Para tentar minimizar infecções por parasitas intestinais, o Plano Nacional de Vigilância e Controle das Enteroparasitoses do Ministério da Saúde visa à definição de estratégias para o controle dessas parasitoses (Tabela 1). 
Tabela 1. Tabela sobre uso correto de medicamentos.

\begin{tabular}{|c|c|c|c|}
\hline Agente & Medicamento & Dose pediátrica & Dose para adulto \\
\hline Ancilostomíase & $\begin{array}{l}\text { Mebendazol } \\
\text { Albendazol }\end{array}$ & $\begin{array}{l}\text { Crianças acima de } \\
\text { dois (02) anos } \\
\text { dosagem a mesma de } \\
\text { adulto. }\end{array}$ & $\begin{array}{c}\text { 100mg, } 2 \text { vezes ao dia, } \\
\text { durante } 3 \text { dias } \\
\text { consecutivos. } \\
\text { 400mg via oral em } \\
\text { dose única. }\end{array}$ \\
\hline Ascaridíase & $\begin{array}{l}\text { Albendazol } \\
\text { Mebendazol }\end{array}$ & $\begin{array}{c}\text { 10mg/Kg, dose única. } \\
1100 \mathrm{mg}, 2 \text { vezes ao dia, } \\
\text { durante } 3 \text { dias } \\
\text { consecutivos. }\end{array}$ & $\begin{array}{l}\text { 400mg/dia, em dose } \\
\text { única para adultos. } \\
\text { 100mg, } 2 \text { vezes ao dia, } \\
\text { durante } 3 \text { dias } \\
\text { consecutivos. }\end{array}$ \\
\hline Amebíase & $\begin{array}{c}\text { Metronidazol } \\
\text { Tinidazol }\end{array}$ & $\begin{array}{c}35 \mathrm{mg} / \mathrm{Kg} / \mathrm{dia} \text {, divididas } \\
\text { em } 3 \text { tomadas, durante } 5 \\
\text { dias. } \\
50 \mathrm{mg} / \mathrm{Kg} / \text { dia. }\end{array}$ & $\begin{array}{c}\text { Metronidazol, 500mg, } \\
3 \text { vezes/dia, durante } 5 \\
\text { dias. } \\
2 \mathrm{~g} \text {, VO, para adultos, } \\
\text { após uma das refeições, } \\
\text { durante } 2 \text { dias. }\end{array}$ \\
\hline Enterobíase & $\begin{array}{l}\text { Albendazol } \\
\text { Mebendazol }\end{array}$ & $\begin{array}{c}\text { 10mg/Kg, VO, dose } \\
\text { única. } \\
\text { 100mg, } 2 \text { vezes ao dia, } \\
\text { durante } 3 \text { dias } \\
\text { consecutivos. }\end{array}$ & $\begin{array}{l}\text { 10mg/Kg, VO, dose } \\
\text { única, até no máximo } \\
\text { de 400mg. } \\
\text { 100mg, } 2 \text { vezes ao dia, } \\
\text { durante } 3 \text { dias } \\
\text { consecutivos. }\end{array}$ \\
\hline Estrongiloidíase & $\begin{array}{l}\text { Ivermectina } \\
\text { Albendazol }\end{array}$ & $\begin{array}{c}\text { Dose única, VO, } \\
\text { obedecendo a escala } \\
\text { de peso corporal } 25 \\
\text { kg: } 1 \text { comp. } \\
\text { 400mg/dia, durante } 3 \\
\text { dias. }\end{array}$ & $\begin{array}{c}\text { Dose única, VO, } \\
\text { obedecendo a escala de } \\
\text { peso corporal } 25 \mathrm{~kg} \text { : } 1 \\
\text { comp. } \\
\text { 400mg/dia, durante } 3 \\
\text { dias. }\end{array}$ \\
\hline
\end{tabular}


A RDC n 98 de 1 de agosto de 2016, dispõe dos critérios de medicamentos que se enquadram como Medicamentos Isentos de Prescrição (MIPs), sendo os antiparasitário pertencentes nessa categoria, por apresentar reações adversas com causalidades conhecidas e reversíveis após a sua suspensão, baixo potencial de interações, período curto de utilização, facilidade de uso pelo paciente e baixo potencial de toxicidade e risco. Os MIPs cumprem com todos os demais fármacos, requisitos de qualidade, segurança e eficácia preconizados pela Legislação Sanitária em vigor (Anvisa, 2016).

Tem-se observado que a Giardia sp. vem apresentando resistência a antiparasitários disponíveis no mercado (Carvalho, 2009). Um estudo realizado por Gupta e Garg (2009) avaliou uma nova forma de tratamento terapêutico, sendo a utilização do Lactobacillus rhamnosus GG, sendo este o primeiro probiótico utilizado na clínica. Os probióticos são considerados microrganismos não patogênicos pertencente ao gênero Lactobacillus e Bifidobacterium, que produzem efeitos terapêuticos benefícios à saúde e bem estar do paciente, se consumido em quantidade adequada, pode aumentar a imunidade do paciente através do reforço da função da barreira da mucosa intestinal, reduzindo a transferência pela mucosa de organismos e metabólitos danosos para o hospedeiro, aumentando a produção de anticorpos na mucosa intestinal, fortalecendo a integridade dos epitélios e o antagonismo direto de microrganismos patogênicos (Ventura et al., 2018). Sugere-se que a dose terapêutica eficaz para benefícios à saúde é de 5 bilhões de unidades formadoras de colônias por dia $\left(5 \times 10^{9}\right.$ UFC/dia) por pelo menos cinco dias (Gupta \& Garg, 2009).

Analisando a prevalência de contaminação por enteroparasitos no presente estudo, foi verificado que os parasitas mais diagnosticados em crianças foram A. lumbricoide, G. lamblia e E. histolytica. Diante dos resultados encontrados, a prevalência de enteroparasitos é um problema socioeconômico, principalmente em regiões subdesenvolvidas e em desenvolvimento, como no caso do Brasil.

\section{Conclusão}

O exame parasitológico de fezes é a melhor forma de diagnóstico, visto que o tratamento deve ser realizado de acordo com cada parasita encontrado. São válidos os incentivos governamentais para a pesquisa e para o desenvolvimento de novas drogas antiparasitárias, preferencialmente, as de amplo espectro. Entretanto, as medidas preventivas de educação sanitária realizadas por professores e pais ainda é uma das principais medidas de se evitar as contaminações por parasitas intestinais em crianças.

De acordo com os achados no presente estudo, sabe-se que as crianças são as mais propícias a adquirirem enteroparasitos, devido à falta de orientação e/ou falta do início do desenvolvimento de hábitos de higiene básica. Neste sentido, mais estudos acerca do assunto são necessários para ocorrer a diminuição dos parasitas intestinais nesta fase.

\section{Referências}

Almeida, A. A., \& Leite, T. S. A. (2020). Entamoeba histolytica como causa da amebíase. Revista Saúde e Meio Ambiente, 10(1), $133-139$.

Andrade, B., Campos, I., Pimenta, J., Santana, L., Alves, N., Chagas, R., Braz, S., Souza, Y., \& de Paula Silva, J. (2019). Práticas educativas sobre medidas profiláticas contra parasitoses e viroses realizadas com crianças no município de Passos-MG. Revista Educação Ambiental em Ação, 68.

Andrade, E. C. D., Leite, I. C. G., Vieira, M. D. T., Abramo, C., Tibiriçá, S. H. C., \& Silva, P. L. (2011). Prevalência de parasitoses intestinais em comunidade quilombola no Município de Bias Fortes, Estado de Minas Gerais, Brasil, 2008. Epidemiologia e Serviços de Saúde, 20 (3), $337-344$.

Antunes, A. S., \& Libardoni, K. S. D. B. (2017). Prevalência de enteroparasitoses em crianças de creches do município de Santo Ângelo, RS. Revista Contexto \& Saúde, 17(32), 144-156.

Agência Nacional de Vigilância Sanitária. ANVISA (2016). Resolução da diretoria colegiada - RDC $N^{\circ} 98$, de $1^{\circ}$ de agosto de 2016. http://portal.anvisa.gov.br/documents/10181/2921766/RDC_98_2016.pdf/32ea4e54-c0ab-459d-903d-8f8a88192412.

Araújo, G. M. S., Walcher, D. L., Previtali, I. F., Lehman, L. M., Costa, M. P., Susin, L. O., Avila, L. F. C., \& Scaini, C. J. (2020). Frequency of enteroparasitic infections and serum positivity for Toxocara spp. in children from a public day care center in Southern Brazil. Brazilian Journal of Biology, 80(2), 305-310. 
Astudillo, O. G., \& Bava, A. J. (2017). Prevalencia de las parasitosis intestinales en el Hospital de Enfermedades Infecciosas "Dr. Francisco Javier Muñiz". Acta bioquímica clínica latinoamericana, 51(4), 681-686.

Auler, M. E., de Almeida Campos, L., Horst, J. A. E., dos Santos, T. B., Miyahara, C. T. S., Paula, C. R., Ruiz, L. S., Gandra, R. F., \& Figueiredo, D. L. A. (2018). Saúde itinerante nos centros municipais de educação infantil do município de Guarapuava-PR; os desafios da promoção da saúde em crianças expostas a doenças parasitárias. Arquivos de Ciências da Saúde da UNIPAR, 22(1).

Batista, T., Trevisol, F. S., \& Trevisol, D. J. (2009). Parasitoses intestinais em pré-escolares matriculados em creche filantrópica no sul de Santa Catarina. Arquivos Catarinenses de Medicina, 38(3).

Berne, A. C., Vieira, J. N., de Avila, L. F. da C., Villela, M. M., Berne, M. E. A., \& Scaini, C. J. (2014). Giardia lamblia: diagnosis using microscopic methods and enzyme-linked immunosorbent assay (ELISA). Revista De Patologia Tropical / Journal of Tropical Pathology, $43(4), 412-419$.

Bragagnollo, G. R., Santos, T. S. D., Fonseca, R. E. P. D., Acrani, M., Branco, M. Z. P. C., \& Ferreira, B. R. (2019). Playful educational intervention with school children on intestinal parasitosis. Revista brasileira de enfermagem, 72(5), 1203-1210.

Brasil. Ministério da Saúde. Secretaria de Vigilância em Saúde. Departamento de Vigilância Epidemiológica. Doenças infecciosas e parasitárias: guia de bolso/ Ministério da Saúde, Secretaria de Vigilância em Saúde, Departamento de Vigilância Epidemiológica. - 8. ed. rev. - Brasília: Ministério da Saúde, 2010. 444 p.: Il. Disponível em:

https://bvsms.saude.gov.br/bvs/publicacoes/doencas_infecciosas_parasitaria_guia_bolso.pdf. Acesso em: 20 de ago. de 2020.

Caldeira, I. P., Sales, I. M. M., Bessa, A. N., Moura, A. C. T. S., Guerra, K. D. O. S., Popoff, D. A. V., D’Angelis, C. E. M., Júnior, G. E. S. G. (2019). Prevalência de parasitas em pacientes atendidos em laboratório de um centro universitário da cidade de Montes Claros, MG. Revista Brasileira de Análises Clínicas (RBAC), 51(3), 234-40.

Cardoso, T. A. E. M., de Leão, M. S., dos Santos Pires, B., de Ávila Antunes, T., Pinto, D. M., Nizoli, L. Q., \& Ferraz, A. (2018). Ocorrência de Giardia spp. em fezes de cães na praia do Laranjal, Pelotas-RS e estudo comparativo entre técnicas. Revista PUBVET, 12, 130.

Carvalho, T. T. R. (2009). Estado atual do conhecimento de Cryptosporidium e Giardia. Revista de Patologia Tropical/Journal of Tropical Pathology, 38(1), 01-16.

Castro, A. A., Bacalhau, F., Ferreira, F., Avillez, C., \& Batalheiro, J. (2019). Entamoeba histolytica como causa de diarreia crônica. Revista Brasileira de Medicina de Família e Comunidade, 14(41), 1917-1917.

Cavalcante, U. M. B., de Melo, S. A. L., \& Lima, C. M. B. L. (2015). Enteroparasitoses na população infantil sua prevalência e os modelos de decisão utilizados: revisão sistemática. Saúde e Pesquisa ISSN 2176-9206, 8(3), 585-590.

Cazorla-Perfetti, D. (2014). Aspectos relevantes de la enterobiosis humana. Revisión crítica. SABER. Revista Multidisciplinaria del Consejo de Investigación de la Universidad de Oriente, 26(3), 221-242.

Centers for Disease Control and Prevention (CDC), 2017. https://www.cdc.gov.

Dall'Orso, P., Cantou, V., Rosano, K., De los Santos, K., \& Giachetto, G. (2014). Ascaris lumbricoides: Complicaciones graves en niños hospitalizados en el Centro Hospitalario Pereira Rossell. Archivos de Pediatría del Uruguay, 85(3), 149-154.

Falavigna, D. L. M., Almeida, A. A. D., Iwazaki, R. S., \& Araújo, S. M. D. (2008). Intestinal parasites in ecotourism region of the state of Paraná, Brazil. Brazilian Archives of Biology and Technology, 51(4), 493-499.

Fernandes, S., Beorlegui, M., Brito, M. J., \& Rocha, G. (2012). Protocolo de parasitoses intestinais. Acta Pediátrica Portuguesa, 43(1), 35-41.

Fonseca, R. E. P., Barbosa, M. C. R., \& Ferreira, B. R. (2017). High prevalence of enteroparasites in children from Ribeirão Preto, São Paulo, Brazil. Revista Brasileira de Enfermagem, 70(3), 566-571.

Fontes, A. M., Gusson, V. P., de Souza, A. A., \& de Souza, M. A. (2017). Identification of enteroparasites in recreation areas of elementary schools in Northern Espírito Santo, Brazil. Revista de Salud Pública, 19, 795-799.

Freitas, C. O. D. (2017). Análise comparativa do uso de medicamentos antiparasitários adquiridos na Unidade Básica de Saúde Santa Cecília Porto Alegre, RS versus drogarias. Trabalho de Conclusão de Curso (Bacharel em Farmácia) - Universidade Federal do Rio Grande do Sul - UFRS.

Gupta, V., \& Garg, R. (2009). Probiotics. Indian journal of medical microbiology, 27(3), 202.

Lacerda, J. S., \& Dias, M. E. (2017). Detecção de Giardia lamblia em exames parasitológicos de fezes: avaliação comparativa de resultados em laboratório de análises clínicas de rede privada e pública. Revista Saúde UniToledo, 1(1), 147-156.

Lacerda, J. S., \& Jardim, C. M. L. (2017). Estudo da prevalência de parasitoses intestinais em pacientes de um laboratório privado de Araçatuba-SP. Revista Saúde UniToledo, 1(1).

Lins, I. V. F., Soares, C. V. D., Costa, R., Soares, G. V. D., Duarte, A. B. S., \& dos Santos Medeiros, J. (2016). Comparação entre o método da fita adesiva e o método de sedimentação espontânea para o diagnóstico de Enterobius vermicularis. Journal of Biology \& Pharmacy and Agricultural Management, 12(4).

Mamus, C. N. C., Moitinho, A. C., Grube, C. C., de Melo, E. M., Weiler, E. B., de Abreu, C. A., Beltrão, L., Soares, P. B., Beltrame, S., Ribeiro, S., \& Aleixo, D. L. (2008). Enteroparasitoses em um centro de educação infantil do município de Iretama/PR. SaBios-Revista de Saúde e Biologia, 3(2).

Muñoz-Antoli, C., Gozalbo, M., Pavón, A., Pérez, P., Toledo, R., \& Esteban, J. G. (2018). Enteroparasites in preschool children on the pacific region of Nicaragua. The American journal of tropical medicine and hygiene, 98(2), 570-575.

Neves, D. P., Melo, A. L., \& Genaro, O. (2011). Parasitologia humana. 12 ed. Rio de Janeiro: Atheneu, 35. 
Research, Society and Development, v. 10, n. 1, e1910111195, 2021 (CC BY 4.0) | ISSN 2525-3409 | DOI: http://dx.doi.org/10.33448/rsd-v10i1.11195

Norberg, A. N., Ribeiro, P. C., Gonçalves, J. S., Sanches, F. G., Silveira, V. F. C., Oliveira, M. F., \& Ferreira, G. G. (2008). Prevalência de ovos, larvas, cistos e oocistos de elementos parasitários em hortaliças comercializadas no município de Nova Iguaçu, Rio de Janeiro, Brasil. Ciência \& Tecnologia, 12.

Pereira, A. S., Shitsuka, D. M., Parreira, F. J., \& Shitsuka, R. (2018). Metodologia da pesquisa científica. [eBook]. Santa Maria.

Serrano, M. L. (2018). Prevenção de parasitose intestinal em crianças menores de cinco anos, Unidade de Saúde do Distrito de Areia Branca II, município de Santana do Ipanema Alagoas. Trabalho de Conclusão de Curso (Especialização Gestão do Cuidado em Saúde da Família) - Universidade Federal de Minas Gerais - UFMG, 2018.

Silva, P. V. D., Maciel, L. D. S., Castro, L. S., Murat, P. G., Higa Junior, M. G., Zerlotti, P. H., Motta-Castro, A. R. C., Pontes, E. R. J. C., Cavalheiros, M. E., \& Dorval, M. E. C. (2018). Enteroparasites in Riverside Settlements in the Pantanal Wetlands Ecosystem. Journal of parasitology research, 2018.

Siqueira, R. L. L. D. S., \& Tristão, T. C. (2019). Ocorrência e geolocalização de parasitos intestinais em laudos parasitológicos de fezes de crianças em um laboratório privado do município de Alto Paraiso/RO nos anos de 2017-2018. Trabalho de Conclusão de Curso (Bacharel em Farmácia) - Faculdade de Educação e Meio Ambiente - FAEMA, 2019.

Solano-Barquero, M., Montero-Salguero, A., León-Alán, D., Santamaría-Ulloa, C., Mora, A. M., \& Reyes-Lizano, L. (2018). Prevalencia de parasitosis en niños de 1 a 7 años en condición de vulnerabilidad en la Región Central Sur de Costa Rica. Acta médica costarricense, 60(2), 19-29.

Teixeira, A. G. S., Santos, F. B., Santos, G. R., de Sousa Santos, M. D. R., \& Rodrigues, G. M. (2018). Os efeitos do saneamento básico precário para o aumento da Ascaris lumbricoides. Revista Brasileira de Pesquisa em Ciências da Saúde, 5(10), 34-40.

Ventura, L. L. A., Oliveira, D. R. D., Gomes, M. A., \& Torres, M. R. F. (2018). Effect of probiotics on giardiasis. Where are we? Brazilian Journal of Pharmaceutical Sciences, 54(2).

Vieira, P. B., Brandelli, C. L. C., Veríssimo, C. D. M., \& Tasca, T. (2012). Mecanismos específicos de patogenicidade de protozoários de mucosa: Entamoeba histolytica, Giardia lamblia e Trichomonas vaginalis. Clinical \& Biomedical Research, 32(1).

Villamizar, X., Higuera, A., Herrera, G., Vasquez-A, L. R., Buitron, L., Muñoz, L. M., Gonzalez-C, F. E., Lopez, M. C., Giraldo, J. C., \& Ramírez, J. D. (2019). Molecular and descriptive epidemiology of intestinal protozoan parasites of children and their pets in Cauca, Colombia: a cross-sectional study. BMC Infectious Diseases, 19(1), 1-11.

Welch, V. A., Hossain, A., Ghogomu, E., Riddle, A., Cousens, S., Gaffey, M., Arora, P., Black, R., Friis, H., Hollingsworth, T. D., \& Chen, L. (2019). Deworming children for soil-transmitted helminth in low and middle-income countries: systematic review and individual participant data network metaanalysis. Journal of development effectiveness, 11(3), 288-306.

Wiebbelling, A. M. P., Corrêa, D. C., Severo, C. B., Schirmer, H., \& Carlesso, A. M. (2019). Prevalência e prevenção de parasitoses intestinais em crianças de creches/escolas de Porto Alegre. Porto Alegre: UFCSPA. 2019.

Zanotto, M., Cavagnolli, N. I., Breda, J. C., Dalla Santa Spada, P. K. W., Bortolini, G. V., \& Rodrigues, A. D. (2018). Prevalence of intestinal parasites and socioeconomic evaluation of a country town in the Serra Gaucha region, Rio Grande do Sul, Brazil. Revista de Patologia Tropical/Journal of Tropical Pathology, 47(1), 19-30.

Zardeto-Sabec, G. (2019). Manual de práticas laboratoriais para o estágio em Análises Clínicas. 1aed. Novas Edições Acadêmicas. 244p.

Zardeto-Sabec, G., Marcon, A. V., Quemel, F. S., Teixeira, D. G., \& Jesus, R. A. (2020). Análise dos laudos do exame parasitológico de fezes de um laboratório da cidade de Umuarama-PR no ano de 2018. Brazilian Journal of Surgery and Clinical Research-BJSCR, 30(3), 07-12. 